\title{
Detection of ammonia in M 51
}

\author{
Shuro Takano ${ }^{1,2, \star}$, Toshiaki Takano ${ }^{3}$, Naomasa Nakai $^{4}$, Kentaro Kawaguchi $^{5}$, and Peter Schilke ${ }^{6}$
}

\author{
1 Nobeyama Radio Observatory ${ }^{\star \star}$, Nobeyama, Minamimaki, Minamisaku, 384-1305 Nagano, Japan \\ e-mail: takano.shuro@nao.ac.jp \\ 2 Department of Astronomical Science, The Graduate University for Advanced Studies (Sokendai), Nobeyama, Minamimaki, \\ Minamisaku, 384-1305 Nagano, Japan \\ 3 Graduate School of Engineering, Chiba University, 1-33 Yayoi-cho, Inage-ku, 263-8522 Chiba, Japan \\ e-mail: takano@faculty.chiba-u.jp \\ 4 Division of Physics, Faculty of Pure and Applied Sciences, University of Tsukuba, 1-1-1 Ten-noudai, Tsukuba, 305-8571 Ibaraki, \\ Japan \\ e-mail: nakai@physics.px.tsukuba.ac.jp \\ 5 Department of Chemistry, Faculty of Science, Okayama University, 3-1-1 Tsushimanaka, 700-8530 Okayama, Japan \\ e-mail: okakent@cc.okayama-u.ac.jp \\ ${ }^{6}$ I. Physikalisches Institut, Universität zu Köln, Zülpicher Straße 77, 50937 Köln, Germany \\ e-mail: schilke@ph1.uni-koeln.de
}

Received 6 December 2011 / Accepted 20 January 2013

\section{ABSTRACT}

\begin{abstract}
Aims. To study the abundance and temperature of ammonia in the center of a nearby galaxy M 51 and to compare them with those in other nearby galaxies, we observed its $(J, K)=(1,1),(2,2),(3,3)$, and $(4,4)$ inversion transitions at the wavelength of $1.3 \mathrm{~cm}$. Methods. The observations were carried out with the Effelsberg 100-m radio telescope.

Results. The $(1,1),(2,2)$, and $(3,3)$ transitions are clearly detected, but the $(4,4)$ transition is barely detected. The rotational temperature obtained from the $(1,1)$ and $(2,2)$ transitions of para-ammonia is $25 \pm 2 \mathrm{~K}$, which is similar to those of $\mathrm{M} 82$ and the Large Magellanic Cloud (N 159 W), but significantly lower than those of IC 342 and NGC 1068 among the nearby galaxies. The column density of ammonia is $(8.1 \pm 2.4) \times 10^{13} \mathrm{~cm}^{-2}$, and the abundance relative to $\mathrm{H}_{2}$ is $\sim 4.5 \times 10^{-9}$. The abundance in M 51 is about a factor of 5 lower than those of NGC 253 and IC 342, but about one order of magnitude higher than those of M 82 and the Large Magellanic Cloud. The addition of the data of M 51 further supports the exceptionally low abundances of ammonia reported previously in these two galaxies. For understanding the abundance of ammonia in M 51 and other nearby galaxies, their temperatures were compared. As a result, we found that the galaxies with low temperature tend to have low abundance of ammonia. In addition, the photodissociation rate of ammonia was compared to those of related molecules detected in the nearby galaxies to discuss the effect of photodissociation. We found that the low abundance of ammonia in some galaxies cannot be explained only by the effect of photodissociation.
\end{abstract}

Key words. local insterstellar matter - molecular processes - ISM: molecules - ISM: abundances

\section{Introduction}

So far, more than 40 molecular species have been detected in external galaxies, mainly through radioastronomical observations. These molecules have been used to study the distributions and kinematics of molecular gas, the physical conditions such as temperature and density, and the chemical reactions.

Ammonia $\left(\mathrm{NH}_{3}\right)$ is a relatively abundant and useful molecule for studying the nature of molecular clouds including kinetic temperature (e.g. Ho \& Townes 1983). It is well observed in Galactic sources and also in several external galaxies.

Ammonia was first detected in external galaxies using the $(J, K)=(1,1)$ inversion transition by Martin \& Ho (1979) in NGC 253 and IC 342. Subsequently, the $(2,2),(3,3)$, and $(4,4)$ lines were detected in IC 342 (Martin et al. 1982; Martin \& Ho 1986). In addition, the spatial distributions of ammonia in IC 342 were mapped with the VLA by Ho \& Martin (1983) and Ho et al. (1990). Ammonia was also observed in the Large Magellanic Cloud (LMC; Ott et al. 2010), other nearby

^ Visiting researcher (2011 June-December): Leiden Observatory, Leiden University, PO Box 9513, 2300 RA Leiden, The Netherlands.

$\star \star$ Nobeyama Radio Observatory is a branch of the National Astronomical Observatory of Japan, National Institutes of Natural Sciences, Japan. galaxies Cen A, Maffei2, M 82, and NGC 1068 (Seaquist \& Bell 1990; Henkel et al. 2000; Takano et al. 2000, 2002; Weiß et al. 2001; Ao et al. 2011), Arp 220 (Takano et al. 2005; Ott et al. 2005, 2011), and more distant sources B0218+357 (Henkel et al. 2005) and PKS1830-211 (Henkel et al. 2008). These data indicate large variations in ammonia abundance among galaxies that are not yet well understood.

M 51 (NGC 5194) is a nearby grand-design spiral galaxy at a distance of 7-8 Mpc (Feldmeier et al. 1997; Takáts \& Vinkó 2006) seen nearly face-on with a low-luminosity active galatic nucleus (AGN; e.g. Fukazawa et al. 2001). Rich molecular gas traced by carbon monoxide $(\mathrm{CO})$ has been mapped in its $J=$ 1-0 and 2-1 transitions with single-dish telescopes (e.g. Nakai et al. 1994; Schuster et al. 2007). High-resolution images with interferometers have also been obtained (e.g. Aalto et al. 1999; Helfer et al. 2003). Recently Koda et al. (2009, 2011) revealed the detailed distribution of $\mathrm{CO}(J=1-0)$ with spatial resolution of 4 " by mosaicing and combining data obtained with Combined Array for Research in Millimeter Astronomy (CARMA) and the Nobeyama 45-m radio telescope.

On the other hand, other limited molecular species except $\mathrm{CO}$ have been detected in M 51, though molecular gas is abundant as mentioned above for the $\mathrm{CO}$ observations. HCN and $\mathrm{HCO}^{+}$were observed including images with interferometers 
(Nguyen et al. 1992; Kohno et al. 1996; Sorai et al. 2002; Schinnerer et al. 2005). CS, $\mathrm{N}_{2} \mathrm{H}^{+}$, and $\mathrm{HNC}$ were detected at the center (Sage et al. 1990; Sage \& Ziurys 1995; Huettemeister et al. 1995). However, the number of detected molecules is rather small compared to other nearby gas-rich starburst galaxies, such as NGC 253, M 82, and IC 342. M 51 has not often been observed to detect molecules, because starburst galaxies were usually observed owing to the relatively strong intensity of the lines. In particular, ammonia has not yet been detected in M 51, although it is fundamental for studying the temperature and the abundance, including their relation to the physical conditions of each galaxy. The temperature and the abundance of ammonia in this spiral galaxy with a low-luminosity AGN will contribute to understanding extragalactic ammonia and its relation to the physical conditions in galaxies.

In this article we report the first detections of the ammonia $(1,1),(2,2)$, and $(3,3)$ lines in M 51. From the present observations we derived the abundance, the rotational temperature, and the ortho-to-para abundance ratio of ammonia. Based on the present study and on knowledge of ammonia in the nearby galaxies studied so far, the temperature and the abundance of ammonia are compared and discussed.

\section{Observations}

The observations were carried out with the 100-m radio telescope of the MPIfR (Max-Planck-Institut für Radioastronomie) at Effelsberg in June 2000 and March 2002. The receiver was equipped with cooled high-electron-mobility transistors (HEMT) for two orthogonal linear polarizations. The four inversion transitions of $(J, K)=(1,1),(2,2),(3,3)$, and $(4,4)$ at the 23.7-24.1 GHz region in both polarizations were observed simultaneously. The $(3,3)$ line $(K$ multiple of 3 ) belongs to orthoammonia, and the other lines to para-ammonia. The spectra were obtained using eight digital spectrometers. The bandwidth of each spectrometer was $40 \mathrm{MHz}$ (512 channels), which corresponds to a velocity coverage of $\sim 506 \mathrm{~km} \mathrm{~s}^{-1}$ at $23.7 \mathrm{GHz}$. The corresponding velocity resolution is $0.99 \mathrm{~km} \mathrm{~s}^{-1}$.

The spatial resolution of the telescope was about $40^{\prime \prime}$ (HPBW) measured at the pointing observations with 3C 286. 3C 286 was also used to check the focus and to calibrate intensity. This resolution corresponds to about $1.6 \mathrm{kpc}$ at the distance of $8 \mathrm{Mpc}$. The adopted central position of M 51 was $\mathrm{RA}(\mathrm{B} 1950.0)=13^{\mathrm{h}} 27^{\mathrm{m}} 46^{\mathrm{s}} .3$ and $\operatorname{Dec}(\mathrm{B} 1950.0)=47^{\circ} 27^{\prime} 10^{\prime} 0$, and the systemic velocity was $V_{\mathrm{LSR}}=470 \mathrm{~km} \mathrm{~s}^{-1}$ in the radio definition (Nakai et al. 1994). The observations were made in the dual-beam-switching mode. The off position was at $121^{\prime \prime}$ in azimuth. The on and off positions were changed by using a rotating horn at the receiver with a switching frequency of $1 \mathrm{~Hz}$.

The data were reduced using the CLASS software ${ }^{1}$. Linear baselines were subtracted from spectra assuming a velocity range of the lines to be between 350 and $550 \mathrm{~km} \mathrm{~s}^{-1}$. In the final spectra ten channels were binned to obtain reduced velocity resolution of $9.9 \mathrm{~km} \mathrm{~s}^{-1}$ at $23.7 \mathrm{GHz}$ and an rms noise of $0.6-1 \mathrm{mK}$.

\section{Results}

The $(J, K)=(1,1),(2,2)$, and $(3,3)$ lines are detected, but the $(4,4)$ line is barely seen as shown in Fig. 1 . The intensity of the $(3,3)$ ortho line is stronger than that of the $(2,2)$ para line as often seen in other sources (e.g., in NGC 253 and NGC 1068, Takano et al. 2002; Mauersberger et al. 2003; Ao et al. 2011). The width of each line (FWHM) is about $120 \mathrm{~km} \mathrm{~s}^{-1}$. This is in good

\footnotetext{
1 http://wWW.iram.fr/IRAMFR/GILDAS
}

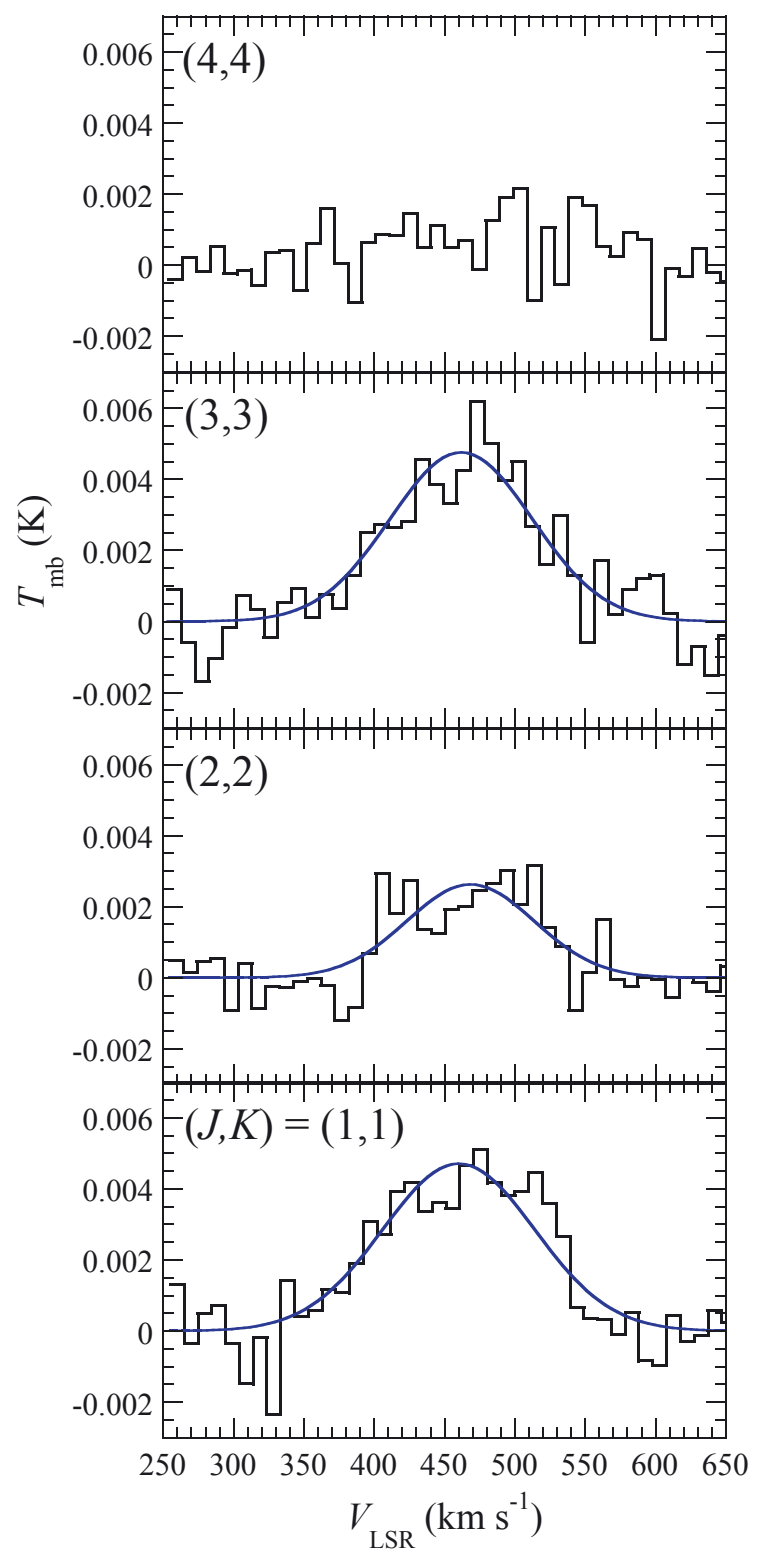

Fig. 1. Spectra of the ammonia $(J, K)=(1,1)$ to $(4,4)$ transitions at the wavelength of $1.3 \mathrm{~cm}$ in M 51. The horizontal axis is velocity with respect to the local standard of rest, calculated from the rest frequency of each transition. The vertical axis shows intensity in the main beam brightness temperature. The spectral channels are binned to a velocity resolution of $9.9 \mathrm{~km} \mathrm{~s}^{-1}$ (see the section of observations).

agreement with the line width measured in HNC $\left(115 \mathrm{~km} \mathrm{~s}^{-1}\right.$, Huettemeister et al. 1995), but slightly narrower than the average CO line profile over the same area $\left(150 \mathrm{~km} \mathrm{~s}^{-1}\right.$, see below). The line parameters are summarized in Table 1.

The obtained data were analyzed with the conventional rotational diagram method (e.g. Turner 1991). The beam filling factor was estimated as follows and was taken into account. The size of the distribution of ammonia was estimated as $\sim 75^{\prime \prime} \times$ $70^{\prime \prime}$ (FWHM) based on the distribution of the CO $J=1-0$ line in the central region (Nakai et al. 1994). By using the wellknown formula (e.g. Burton et al. 1992) for the coupling between a two-dimensional Gaussian distributed source and the telescope beam, the beam-filling factor was calculated as 0.77 . The partition function was calculated by numerical summation of populations in the metastable levels $(J=K)$, where populations are dominated. 
Table 1. Observed line parameters of ammonia in M 51.

\begin{tabular}{lcccccc}
\hline \hline Transition & $\begin{array}{c}\int T_{\mathrm{mb}} \mathrm{d} v^{1} \\
\left(\mathrm{~K} \mathrm{~km} \mathrm{~s}^{-1}\right)\end{array}$ & $\begin{array}{c}T_{\mathrm{mb}}^{2} \\
(\mathrm{mK})\end{array}$ & $\begin{array}{c}V_{\mathrm{LSR}}^{2} \\
\left(\mathrm{~km} \mathrm{~s}^{-1}\right)\end{array}$ & $\begin{array}{c}\Delta v^{2} \\
\left(\mathrm{~km} \mathrm{~s}^{-1}\right)\end{array}$ & $\begin{array}{c}\text { rms noise } \\
(\mathrm{mK})\end{array}$ & $\begin{array}{c}N(J, K)^{3} \\
\left(\mathrm{~cm}^{-2}\right)\end{array}$ \\
\hline$(1,1)$ & $0.61 \pm 0.04$ & 4.7 & 460 & 127 & 1 & $(1.0 \pm 0.1) \times 10^{13}$ \\
$(2,2)$ & $0.27 \pm 0.03$ & 2.6 & 468 & 107 & 0.6 & $(3.4 \pm 0.3) \times 10^{12}$ \\
$(3,3)$ & $0.56 \pm 0.04$ & 4.8 & 462 & 119 & 1 & $(6.3 \pm 0.5) \times 10^{12}$ \\
$(4,4)$ & $(0.16 \pm 0.03)^{4}$ & $\ldots$ & $\ldots$ & $\ldots$ & 0.6 & $<(1.7 \pm 0.3) \times 10^{12}$ \\
\hline
\end{tabular}

Notes. ${ }^{(1)}$ Obtained by integrating the spectra between $350-550 \mathrm{~km} \mathrm{~s}^{-1}$. The error was estimated from the noise level of each spectrum. ${ }^{(2)}$ Obtained from Gaussian fits to the spectra. ${ }^{(3)}$ Column density of each level. ${ }^{(4)}$ Barely detected.

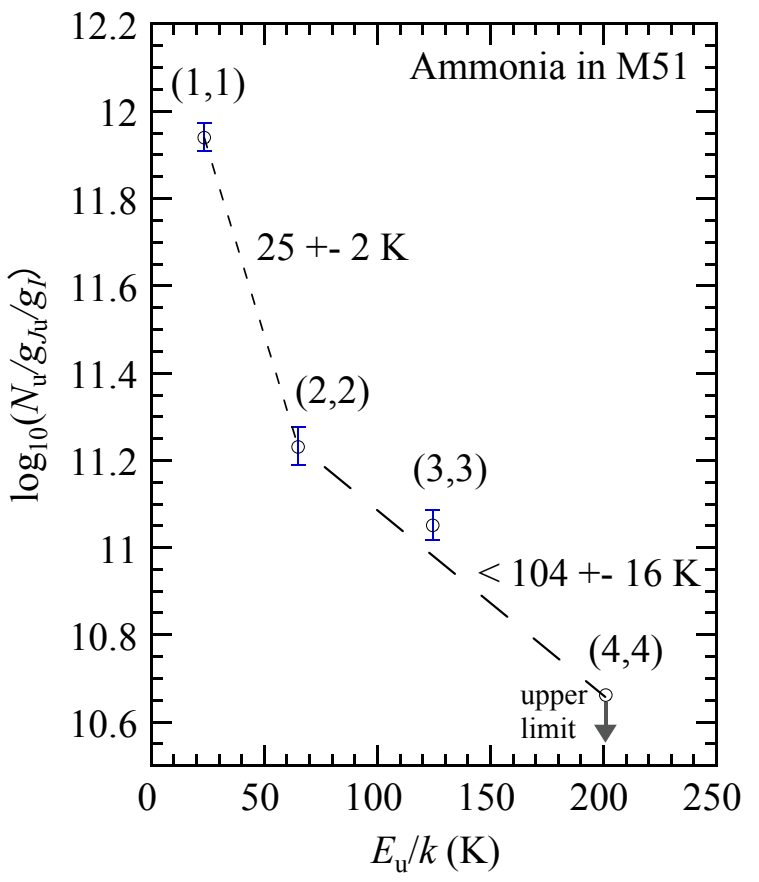

Fig. 2. Rotational diagram of ammonia in M51. The horizontal axis is the upper state energy, and the vertical axis corresponds to the abundances $\left(g_{J_{\mathrm{u}}}=2 J_{\text {upper }}+1, g_{I}\right.$ is the spin statistical weight $)$. The $(4,4)$ line is regarded as an upper limit, because it is barely detected. The error of each data point was estimated based on the noise level of each spectrum. About the rotational diagram analysis, see the section of results and references there.

The diagram is shown in Fig. 2. The rotational (kinetic) temperature obtained is $25 \pm 2 \mathrm{~K}$ based on the detected para lines, $(1,1)$ and $(2,2)$. The $(4,4)$ line is barely detected as mentioned before. However, if the detection is real, the data point will lie at a much higher position than the extrapolated dashed line connecting the two data points of the $(1,1)$ and $(2,2)$ lines. This would imply the existence of a hotter gas component than $25 \mathrm{~K}$. (The temperature calculated using the $(2,2)$ line and the $(4,4)$ upper limit is $<104 \pm 16 \mathrm{~K}$ as shown in Fig. 2.) The temperature obtained from the $(1,1)$ and $(2,2)$ lines is thought to represent relatively low-excitation gas. The data point of the $(3,3)$ ortho line also lies above the extrapolated dashed line, suggesting a high ortho-to-para abundance ratio.

The column densities obtained are $(1.4 \pm 0.2) \times 10^{13} \mathrm{~cm}^{-2}$ for para-ammonia based on the $(1,1)$ and $(2,2)$ transitions, and $(6.7 \pm 2.4) \times 10^{13} \mathrm{~cm}^{-2}$ for ortho-ammonia based on the $(3,3)$ transition, where the rotational temperature of ortho-ammonia was assumed to be the same as that of para-ammonia. The total column density (ortho + para) is $(8.1 \pm 2.4) \times 10^{13} \mathrm{~cm}^{-2}$. The abundance relative to the column density of $\mathrm{H}_{2}$ is $(4.5 \pm 1.3) \times 10^{-9}$, where the column density of
$\mathrm{H}_{2}\left(1.8 \times 10^{22} \mathrm{~cm}^{-2}\right)$ was estimated from the CO integrated intensity ( $I_{\mathrm{CO}}$; Nakai et al. 1994, beam size $\left.16^{\prime \prime}\right)$ averaged within the $40^{\prime \prime}$ beam of our observations and the conversion factor $\left(N\left(\mathrm{H}_{2}\right)=2 \times 10^{20} I_{\mathrm{CO}} \mathrm{cm}^{-2}\right)$ in Nakai et al. (1994).

To check the validity of using the $\mathrm{CO}$ line for the purpose above, we investigated the CO linewidth (Nakai et al. 1994; Kuno \& Nakai 1997) by direct comparison and by convolving their CO map with the beam size of our observations. As a result, the maximum linewidth in the central region is $155 \mathrm{~km} \mathrm{~s}^{-1}$ (FWHM), and the linewidth from the convolved image is about $150 \mathrm{~km} \mathrm{~s}^{-1}$ (FWHM). These widths are about 1.2-1.4 times larger than those of ammonia suggesting that $\mathrm{CO}$ is also probing kinematics of lower density regions. However, the difference in the linewidth above is not so large as to lead to a significant effect on the abundance of ammonia averaged within the beam.

The ortho-to-para abundance ratio is found to be $4.8 \pm 1.0$. This value is based on only one ortho line $(3,3)$, and thus it is not possible to determine the rotational temperature of orthoammonia. Future observations of another ortho line $(6,6)$ and para lines $(4,4)$ and $(5,5)$ will be useful to obtain an accurate ratio. The ratio is much higher than the value of one at a hightemperature limit, but similar to what is found in NGC 253 (velocity range: $100-400 \mathrm{~km} \mathrm{~s}^{-1}$ ) (Takano et al. 2002).

\section{Discussion}

\subsection{Rotational temperature of ammonia}

The rotational temperature obtained in M $51(25 \pm 2 \mathrm{~K}$ from the $(1,1)$ and $(2,2)$ para lines) is compared to those obtained in other nearby galaxies and the central molecular zone (CMZ) of our Galaxy. The temperatures mainly obtained from the $(1,1)$ and $(2,2)$ para lines are listed in Table 2 . As shown in this table, the temperature in M 51 is similar to those in M 82 and the CMZ, but significantly lower than those in IC 342 and NGC 1068. These results indicate that the gas in M51 averaged over the beam is less heated than in IC 342 and NGC 1068. To interpret this situation, two factors can be pointed out.

(1) The gas may actually be less heated than in IC 342 and NGC 1068. This should come from star formation and/or AGN activities. The star formation rate (SFR) of some nearby galaxies are listed in Smith \& Wilson (2003), and as a result, the SFR of M $51\left(0.9 M_{\odot} \mathrm{yr}^{-1}\right)$ is about a factor of 5 lower than that of NGC $1068\left(5.0 M_{\odot} \mathrm{yr}^{-1}\right)$. It is probable that this difference is related to the lower temperature in M 51.

The dust temperature in M51 (34.8 K) is relatively low among nearby galaxies (e.g. $46.3 \mathrm{~K}$ in NGC 1068), but it is also low in IC 342 (34.9 K) (Young et al. 1989). In addition, infrared luminosity in M $51\left(5 \times 10^{9} L_{\odot}\right)$ is relatively low among nearby galaxies (e.g. $3.2 \times 10^{11} L_{\odot}$ in NGC 1068$)$, but it is also low in IC $342\left(3.8 \times 10^{9} L_{\odot}\right)$ (Thronson et al. 1987). 
A\&A 552, A34 (2013)

Table 2. Ammonia abundance and temperature in nearby galaxies and the Galactic center.

\begin{tabular}{|c|c|c|c|c|}
\hline Galaxy & $\begin{array}{c}\text { Temperature }^{1} \\
(\mathrm{~K})\end{array}$ & Abundance & $\begin{array}{l}\text { Spatial resolution } \\
\text { (arcsec and } \mathrm{kpc})\end{array}$ & Reference \\
\hline M51 & $25 \pm 2$ & $(4.5 \pm 1.3) \times 10^{-9}$ & $40^{\prime \prime}, 1.6$ (Effelsberg) & this work \\
\hline NGC 253 & $\begin{array}{c}29(+7,-5), 50(+17,-10)^{2} \\
\ldots\end{array}$ & $\begin{array}{c}(2.7 \pm 1.1) \times 10^{-8} \\
2.0 \times 10^{-8}\end{array}$ & $\begin{array}{c}71^{\prime \prime}, 1.0(\mathrm{NRO}) \\
40^{\prime \prime}, 0.48 \text { (Effelsberg) }\end{array}$ & $\begin{array}{c}\text { Takano et al. (2002) } \\
\text { Mauersberger et al. (2003) }\end{array}$ \\
\hline M 82 & $29 \pm 5$ & $5 \times 10^{-10}$ & $40^{\prime \prime}, 0.75$ (Effelsberg) $^{3,4}$ & Weiß et al. (2001) \\
\hline IC 342 & $57 \pm 1$ & $1.9 \times 10^{-8}$ & $40^{\prime \prime}, 0.34$ (Effelsberg) $^{3,4}$ & Takano et al. (2002) \\
\hline & $53 \pm 1$ & $1.3 \times 10^{-8}$ & $40^{\prime \prime}, 0.34$ (Effelsberg) $^{3}$ & Mauersberger et al. (2003) \\
\hline Maffei 2 & $\begin{array}{c}30(+3,-2) \\
\quad 48 \pm 15\end{array}$ & $\begin{array}{c}1 \times 10^{-7} \\
2.0 \times 10^{-8}\end{array}$ & $\begin{array}{c}70^{\prime \prime}, 1.7(\mathrm{NRO}) \\
40^{\prime \prime}, 0.48(\text { Effelsberg })^{3}\end{array}$ & $\begin{array}{c}\text { Takano et al. (2000) } \\
\text { Mauersberger et al. (2003) }\end{array}$ \\
\hline NGC 1068 & $44(+6,-4)$ & $(2.9 \pm 0.6) \times 10^{-8}$ & $31^{\prime \prime}, 2.3(\mathrm{GBT})$ & Ao et al. (2011) \\
\hline $\mathrm{LMC}(\mathrm{N} 159 \mathrm{~W})$ & $\sim 16$ & $4 \times 10^{-10}$ & $18^{\prime \prime} 6 \times 15^{\prime \prime} .7, \sim 0.004$ (ATCA) & Ott et al. (2010) \\
\hline Galactic center (CMZ) & $\sim 20-40^{5}$ & $1 \times 10^{-9}$ (fixed) & 570", 0.024 (Kagoshima) & Nagayama et al. (2007) \\
\hline
\end{tabular}

Notes. ${ }^{(1)}$ Rotational temperature obtained mainly from the $(1,1)$ and $(2,2)$ para-ammonia transitions. ${ }^{(2)}$ At the velocity component of 260-320 $\mathrm{km} \mathrm{s}^{-1}$. (3) The original linear scale in Table 4 in Mauersberger et al. (2003) corresponds to the angular resolution of $35^{\prime \prime}$. The linear scale here was converted for $40^{\prime \prime} .{ }^{(4)}$ Taken from Mauersberger et al. $(2003) .{ }^{(5)} \sim 75 \%$ of the total flux of ammonia corresponds to this temperature range.

These facts indicate that the direct comparisons among these values are not appropriate. Observations with higher spatial resolution and comparisons with similar angular resolution are desirable for better understanding the temperature.

(2) The ratio of cold gas relative to hot gas in the beam $(1.6 \mathrm{kpc}$ at $D=8 \mathrm{Mpc}$ ) may be higher in M 51. Devereux \& Young (1992) reported that the $\sim 90-94 \%$ of the dust mass in M 51 is in cold temperatures of $\sim 14-16 \mathrm{~K}$, based on the observational data at $170 \mu \mathrm{m}$. This result supports this second point.

The rotational temperatures obtained from the ammonia inversion lines correspond to the kinetic temperatures, but generally they underestimate the kinetic temperatures, in particular at higher temperatures (Walmsley \& Ungerechts 1983; Danby et al. 1988). The obtained rotational temperature of $25 \pm 2 \mathrm{~K}$ for M 51 corresponds to about $33 \mathrm{~K}$ in kinetic temperature according to the Fig. 1 in Danby et al. (1988).

\subsection{Abundance of ammonia}

The abundances of ammonia in nearby galaxies are also summarized in Table 2 and Fig. 3. The abundance in M51 is about a factor of 5 lower than those in NGC 253 and IC 342, but about one order of magnitude higher than those in M 82 and the LMC (N $159 \mathrm{~W})$. The significantly low abundances of ammonia in M 82 (Weiß et al. 2001; Takano et al. 2002) and in the LMC (Ott et al. 2010) have already been pointed out.

In the present study, the addition of the data of M 51 further supports the exceptionally low abundances of ammonia in M 82 and the LMC. In addition, the data of M 51 may indicate similar characteristics to M82 and the LMC, since the abundance in M51 is halfway between the ammonia-rich galaxies and the ammonia-poor galaxies. A possible common characteristic of galaxies (including M 51) with low ammonia abundance is to have relatively low temperature $(\leq 29 \mathrm{~K}$, Table 2$)$. The abundance of ammonia in gas phase is thought to increase in active regions (see compilation and references in Takano et al. 2002). If it is produced in gas phase with ion-molecule reactions, a low activation energy must be overcome. If it is produced on a grain surface (e.g. recent experiment, Hidaka et al. 2011), it must be subsequently evaporated to gas phase. The above common characteristic is consistent with these formation mechanisms, because the ion-molecule formation reaction and the evaporation of ammonia are both not efficient at low temperatures. In the case

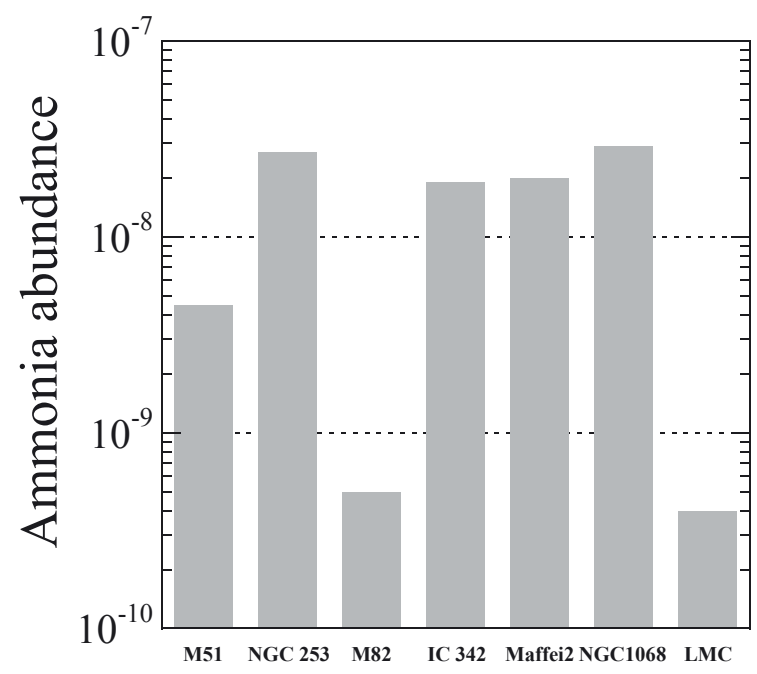

Fig. 3. Abundance of ammonia relative to $\mathrm{H}_{2}$ in nearby galaxies. The data were taken from the references as follows (see also Table 2): NGC 253 Takano et al. (2002), M 82 Weiß et al. (2001), IC 342 Takano et al. (2002), Maffei 2 Mauersberger et al. (2003), NGC 1068 Ao et al. (2011), and LMC (N 159 W) Ott et al. (2010).

of the LMC, the low metallicity $(\sim 10 \%$ of the solar abundance for nitrogen, Hunter et al. 2007) should also make the abundance of ammonia lower (Ott et al. 2010).

On the other hand, the reason for the low abundances of ammonia in M 82 and the LMC has been discussed as being due to the efficient photodissociation of ammonia, in particular in M 82 (Weiß et al. 2001). In addition, we suggested previously that the formation process of ammonia on grain surfaces in M 82 is not very efficient based on the list of molecules deficient in $\mathrm{M} 82\left(\mathrm{SiO}, \mathrm{NH}_{3}, \mathrm{HNCO}, \mathrm{CH}_{3} \mathrm{CN}\right.$, and $\left.\mathrm{CH}_{3} \mathrm{OH}\right)$ among nearby galaxies (Takano et al. 2002). Recently, Aladro et al. (2011) have reported results of a molecular line survey of M 82 at the wavelengths of 1.3 and $2 \mathrm{~mm}$, and compared the molecular abundances in M 82 and NGC 253 in detail. Based on this comparison they also point out the existence of a larger amount of gas affected by photodissociation in M 82 .

We compared the photodissociation rates of related molecules in the standard interstellar radiation field (van Dishoeck et al. 2006). The rate of ammonia of $1.2 \times$ $10^{-9} \mathrm{~s}^{-1}$ is a relatively high value and is similar to those 
of molecules deficient in M $82\left(1.6 \times 10^{-9} \mathrm{~s}^{-1}\right.$ for $\mathrm{SiO}$ and $1.4 \times 10^{-9} \mathrm{~s}^{-1}$ for $\left.\mathrm{CH}_{3} \mathrm{OH}\right)$. However, the photodissociation rates of some molecules with comparable or higher abundances in $\mathrm{M} 82$ than in $\mathrm{NGC} 253$ ( $\mathrm{SO}, \mathrm{HCO}, \mathrm{H}_{2} \mathrm{~S}, \mathrm{H}_{2} \mathrm{CO}$, and cyclic- $\left.\mathrm{C}_{3} \mathrm{H}_{2}\right)$ (Aladro et al. 2011) are similary high $\left(4.2 \times 10^{-9}\right.$, $1.1 \times 10^{-9}, 3.1 \times 10^{-9}, 1.0 \times 10^{-9}$, and $1.9 \times 10^{-9} \mathrm{~s}^{-1}$, respectively). These results indicate that the effect of photodissociation in M82 is not always dominant for molecules with high photodissociation rates, though photodissociation is one of the important destruction mechanisms. As usual, balances of both formation and destruction (including photodissociation) mechanisms are important for understanding the molecular abundances. Such considerations are also needed to understand the abundance of ammonia in nearby galaxies. As a formation mechanism in this case, we already mentioned above the possibility of inefficiency of grain surface reactions (Takano et al. 2002) and evaporation to gas phase.

\subsection{Ortho-to-para abundance ratio of ammonia}

If the present value of $4.8 \pm 1.0$ is regarded as fixed at the time of evaporation from grain mantles, the corresponding temperature is $\sim 9 \mathrm{~K}$ (e.g. Takano et al. 2002). We note that the ortho $(0,0)$ level is situated at the lowest energy, so that the large amount of ortho-ammonia is populated there. The population of this $(0,0)$ level is calculated by extrapolating the $(3,3)$ data using the rotational temperature. We assumed the same rotational temperature for ortho-ammonia as for para-ammonia. If the rotational temperature of ortho-ammonia is higher than for para-ammonia, the present column density of ortho-ammonia is overestimated. The column density of ortho-ammonia and the corresponding ortho-to-para abundance ratio are calculated for possible higher rotational temperatures of ortho-ammonia: $3.5 \times 10^{13} \mathrm{~cm}^{-2}$ and 2.5 at $30 \mathrm{~K}, 1.6 \times 10^{13} \mathrm{~cm}^{-2}$ and 1.2 at $40 \mathrm{~K}$, and $1.2 \times 10^{13} \mathrm{~cm}^{-2}$ and 0.8 at $50 \mathrm{~K}$. The ratio is, therefore, largely affected by the variation in rotational temperature.

\section{Concluding remarks}

We reported our detections of the ammonia $(J, K)=(1,1)$, $(2,2)$, and $(3,3)$ lines in the center of a nearby galaxy M 51. The rotational temperature obtained from the para-ammonia lines is $25 \pm 2 \mathrm{~K}$, which is relatively low among the nearby galaxies. The abundance of ammonia in M51 is halfway between the ammonia-rich galaxies (e.g. NGC 253 and IC 342) and the ammonia-poor galaxies (M 82 and the LMC). A possible common characteristic of galaxies (including M 51) with low ammonia abundance is relatively low temperature, which can cause inefficient formation of ammonia in the gas phase and/or inefficient evaporation from the grain surface.

The photodissociation rate of ammonia is relatively high. Therefore, it has been suggested that the low abundance of ammonia in M 82 and the LMC is due to efficient photodissociation by ultraviolet radiation. However, we mentioned that considerations of both the formation and destruction mechanisms are needed, as usual, to understand the abundance of ammonia. This is based on comparing the photodissociation rates of related molecules in nearby galaxies.

Further observations of the high- $(J, K)$ lines will be useful for precise determinations of the temperature, the abundance, and the ortho-to-para ratio. In addition, observations with high spatial resolution will also be useful for understanding spatial variations of the excitation and the abundance.
Acknowledgements. We thank the anonymous referee for valuable comments. We thank C. Henkel, F. Wyrowski, and staff members at Effelsberg for their kind support during the observations. We thank W. J. Altenhoff for valuable discussion of the interpretation of the intensity of the calibrators. S.T. thanks Leiden Observatory for hospitality during his stay. S.T. is grateful to E.F. van Dishoeck for discussions and comments on the manuscript, to G. Winnewisser and R. Kawabe for encouragement, and to the Yamada Science Foundation, Netherlands Research School for Astronomy (NOVA), and the Foundation for Promotion of Astronomy for financial support.

\section{References}

Aalto, S., Hüttemeister, S., Scoville, N. Z., \& Thaddeus, P. 1999, ApJ, 522, 165 Aladro, R., Martín, S., Martín-Pintado, J., et al. 2011, A\&A, 535, A84

Ao, Y., Henkel, C., Braatz, J. A., et al. 2011, A\&A, 529, A154

Burton, W. B., Elmegreen, B. G., \& Genzel, R. 1992, The galactic interstellar medium

Danby, G., Flower, D. R., Valiron, P., Schilke, P., \& Walmsley, C. M. 1988, MNRAS, 235, 229

Devereux, N. A., \& Young, J. S. 1992, AJ, 103, 1536

Feldmeier, J. J., Ciardullo, R., \& Jacoby, G. H. 1997, ApJ, 479, 231

Fukazawa, Y., Iyomoto, N., Kubota, A., Matsumoto, Y., \& Makishima, K. 2001, A\&A, 374, 73

Helfer, T. T., Thornley, M. D., Regan, M. W., et al. 2003, ApJS, 145, 259

Henkel, C., Mauersberger, R., Peck, A. B., Falcke, H., \& Hagiwara, Y. 2000, A\&A, 361, L45

Henkel, C., Jethava, N., Kraus, A., et al. 2005, A\&A, 440, 893

Henkel, C., Braatz, J. A., Menten, K. M., \& Ott, J. 2008, A\&A, 485, 451

Hidaka, H., Watanabe, M., Kouchi, A., \& Watanabe, N. 2011, Physical Chemistry Chemical Physics (Incorporating Faraday Transactions), 13, 15798

Ho, P. T. P., \& Martin, R. N. 1983, ApJ, 272, 484

Ho, P. T. P., \& Townes, C. H. 1983, ARA\&A, 21, 239

Ho, P. T. P., Martin, R. N., Turner, J. L., \& Jackson, J. M. 1990, ApJ, 355, L19 Huettemeister, S., Henkel, C., Mauersberger, R., et al. 1995, A\&A, 295, 571

Hunter, I., Dufton, P. L., Smartt, S. J., et al. 2007, A\&A, 466, 277

Koda, J., Scoville, N., Sawada, T., et al. 2009, ApJ, 700, L132

Koda, J., Sawada, T., Wright, M. C. H., et al. 2011, ApJS, 193, 19

Kohno, K., Kawabe, R., Tosaki, T., \& Okumura, S. K. 1996, ApJ, 461, L29

Kuno, N., \& Nakai, N. 1997, PASJ, 49, 279

Martin, R. N., \& Ho, P. T. P. 1979, A\&A, 74, L7

Martin, R. N., \& Ho, P. T. P. 1986, ApJ, 308, L7

Martin, R. N., Ruf, K., \& Ho, P. T. P. 1982, Nature, 296, 632

Mauersberger, R., Henkel, C., Weiß, A., Peck, A. B., \& Hagiwara, Y. 2003, A\&A, 403, 561

Nagayama, T., Omodaka, T., Handa, T., et al. 2007, PASJ, 59, 869

Nakai, N., Kuno, N., Handa, T., \& Sofue, Y. 1994, PASJ, 46, 527

Nguyen, Q.-R., Jackson, J. M., Henkel, C., Truong, B., \& Mauersberger, R. 1992, ApJ, 399, 521

Ott, J., Weiß, A., Henkel, C., \& Walter, F. 2005, in The Evolution of Starbursts, eds. S. Hüttmeister, E. Manthey, D. Bomans, \& K. Weis, AIP Conf. Ser., 783, 141

Ott, J., Henkel, C., Staveley-Smith, L., \& Weiß, A. 2010, ApJ, 710, 105

Ott, J., Henkel, C., Braatz, J. A., \& Weiß, A. 2011, ApJ, 742, 95

Sage, L. J., \& Ziurys, L. M. 1995, ApJ, 447, 625

Sage, L. J., Shore, S. N., \& Solomon, P. M. 1990, ApJ, 351, 422

Schinnerer, E., Weiss, A., Aalto, S., et al. 2005, in Starbursts: From 30 Doradus to Lyman Break Galaxies, eds. R. de Grijs, \& R. M. González Delgado (Astrophysics and Space Science Library), 329, 251

Schuster, K. F., Kramer, C., Hitschfeld, M., Garcia-Burillo, S., \& Mookerjea, B. 2007, A\&A, 461, 143

Seaquist, E. R., \& Bell, M. B. 1990, ApJ, 364, 94

Smith, D. A., \& Wilson, A. S. 2003, ApJ, 591, 138

Sorai, K., Nakai, N., Kuno, N., \& Nishiyama, K. 2002, PASJ, 54, 179

Takano, S., Nakai, N., Kawaguchi, K., \& Takano, T. 2000, PASJ, 52, L67

Takano, S., Nakai, N., \& Kawaguchi, K. 2002, PASJ, 54, 195

Takano, S., Nakanishi, K., Nakai, N., \& Takano, T. 2005, PASJ, 57, L29

Takáts, K., \& Vinkó, J. 2006, MNRAS, 372, 1735

Thronson, Jr., H. A., Walker, C. K., Walker, C. E., \& Maloney, P. 1987, ApJ, 318,645

Turner, B. E. 1991, ApJS, 76, 617

van Dishoeck, E. F., Jonkheid, B., \& van Hemert, M. C. 2006, Faraday Discussions, 133, 231

Walmsley, C. M., \& Ungerechts, H. 1983, A\&A, 122, 164

Weiß, A., Neininger, N., Henkel, C., Stutzki, J., \& Klein, U. 2001, ApJ, 554, L143

Young, J. S., Xie, S., Kenney, J. D. P., \& Rice, W. L. 1989, ApJS, 70, 699 